\title{
Inspection of Large Droplets in Fuel Sprays Using a Gaussian Laser-Sheet: An Experimental Approach
}

\author{
Rami Zakaria \\ The University of Warwick, School of Engineering, Coventry CV4 7AL, UK \\ Correspondence should be addressed to Rami Zakaria, eng.zak@gmail.com
}

Received 20 December 2011; Accepted 14 February 2012

Academic Editors: Y. Cai, D.-N. Wang, and D. H. Woo

Copyright ( 2012 Rami Zakaria. This is an open access article distributed under the Creative Commons Attribution License, which permits unrestricted use, distribution, and reproduction in any medium, provided the original work is properly cited.

\begin{abstract}
A laser sheet imaging system was developed for the investigation of high-speed fuel sprays under a relatively low injection pressure (less than $1 \mathrm{MPa}$ ). A pulsed laser and high-resolution CCD cameras were used for the evaluation of the fuel injection system of a small IC engine. Large droplets were detected during the injection incident, with a variation in the scattered light pattern from one droplet to another. The light scattering pattern of individual droplets was investigated in order to study the interaction between the laser beam and large fluid droplets compared to the wavelength. A laser sheet, with a wide waist Gaussian profile, was used for illumination, so that the relative position of a droplet in the third dimension of the field of view (FOV) can be estimated from the 2D-image. Light scattering images were processed in order to closely investigate the structure of the fuel droplets, and the behaviour of the laser beam when encountering large droplets $(0.2-1.4 \mathrm{~mm})$. The Particle Image Velocimetry (PIV) method was applied on the double exposure spray images to calculate the droplet velocity distribution of the global spray, using a high temporal resolution $(15 \mu \mathrm{s})$.
\end{abstract}

\section{Introduction}

The fluid atomisation topic is of importance to a wide range of engineering applications, including internal combustion engines. Increasing the overall surface area of the fuel is essential for the improvement of the chemical reaction rate and the volumetric efficiency of the combustion process. This can be done by producing small fuel droplets in the micrometer range. Optical diagnostics have become popular in experimental fluid dynamics due to their nonintrusive nature and high accuracy, thanks to the high temporal and spatial resolution of these methods. The currently available lasers can produce ultra-short illumination pulses, so that measurements can be done on the particle size, density, and velocity, using digital imaging techniques. The term "large droplets" is used here to refer to fluid drops much larger than the wavelength of the light source. The diffractionbased particle sizing techniques, such as phase doppler anemometry (PDA), need a continuous spray flow, and cannot be practically applied on the investigation of individual droplets [1]. Since PDA relies mainly on the Mie solution of the light scattering problem, the obvious limitation of this technique is that the droplets are always considered perfectly spherical. If no external forces are applied on a certain amount of liquid, the surface tension force shapes out the liquid into a compact spherical form, as this has the minimum possible surface energy. Liquid instability is mainly controlled by the dimensionless Weber number [13], which can be given by the relation:

$$
\mathrm{We}=\frac{\rho_{g} \cdot U_{l}^{2} \cdot D}{\sigma} .
$$

The droplet sphericity assumption is acceptable when dealing with fluids at low Weber numbers; that is, for very small droplets, moving at a low relative velocity (between the fluidic phase and the gaseous phase). In this case, the droplets can be assumed stable at their spherical mode for a long period of time. The critical Weber number, at which a hydrocarbon liquid fuel starts atomising, is usually between 10 and 12. A fuel droplet with a $0.5 \mathrm{~mm}$ diameter, for example, reaches its critical Weber number when travels at a relative velocity higher than $22-25 \mathrm{~ms}^{-1}$.

The conventional fuel injectors usually use a high injection pressure behind the nozzle (exceeding $150 \mathrm{MPa}$ in DI 


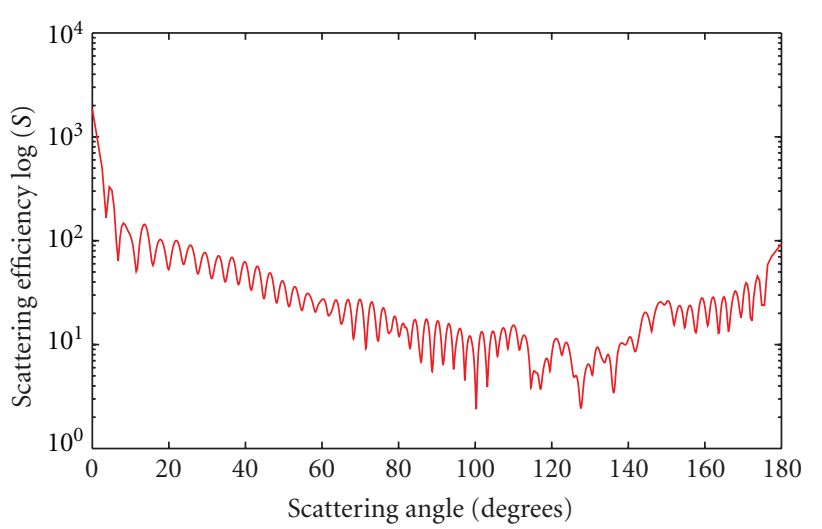

(a)

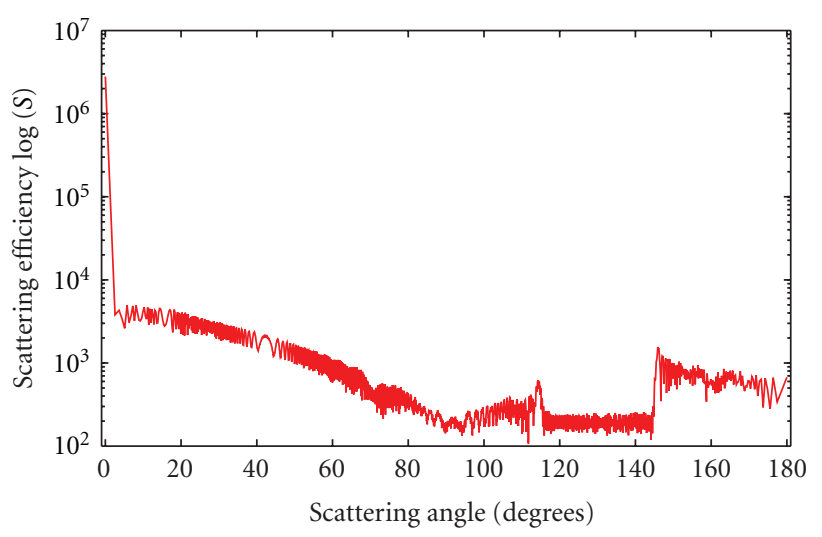

(b)

FIGURE 1: Simulation of the Mie scattering using (a) 10 microns and (b) 400 microns kerosene droplets for green laser beam $(532 \mathrm{~nm})$.

engines [4]) in order to produce a relatively high discharge velocity. The purpose of the high velocity is to produce an early breakup of the fluid during the injection event. However, producing such a high pressure is not possible in the small engines, which are essentially designed for high power-to-weight ratio applications. Power consumption and engine weight are crucial factors in this case, besides the low fuel delivery rate required to operate this type of engine. Fuel injection using low pressure rates produces sprays less homogeneous than the conventional diesel and gasoline injectors, and thus, a wide spectrum of droplet diameters is expected in this case. Because of the low Weber number at the moment of fluid discharge, large fluid ligaments are produced during the fluid fragmentation process, which eventually turn into large droplets mostly concentrated in the central part of the spray jet. Such droplets are also produced by the atomiser during the very beginning stage of the injection incident, due to either the liquid remains inside the swirl chamber of the injector, or the fluid segments attached to the injector nozzle. In both cases, when an injection event occurs, large drops with a low radial velocity are released from the nozzle.

The light scattering mechanism of small particles is explained by the well-established Mie theory (after Gustav Mie, published in 1908). The Mie theory provides a solution for Maxwell's wave equations of a plane electromagnetic

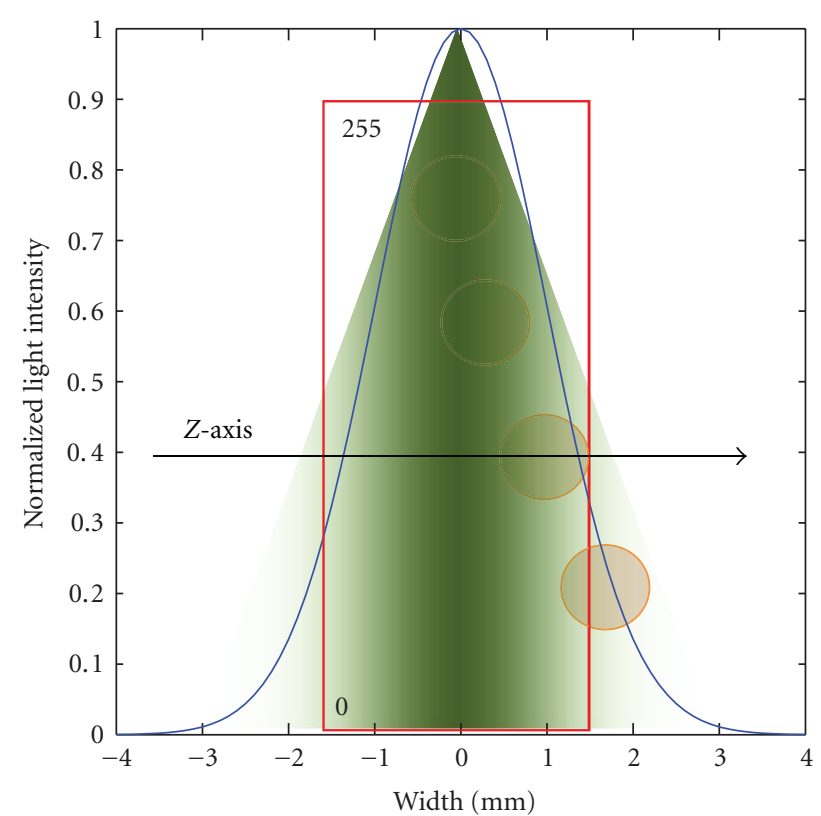

FIGURE 2: The Gaussian profile of the laser sheet and the camerasensitivity area; demonstrating a particle position within the laser sheet.

wave scattered by a homogeneous sphere [5]. The Mie scattering pattern shows a dominant forward lobe for the large particles due to the light diffraction, while the small particles (within the Rayleigh diffraction range) generate a more isotropic scattering pattern and show stronger scattering power at shorter wavelengths $[6,7]$. Figure 1 shows a computer simulation of the Mie scattering pattern using kerosene droplets, 10 microns and 400 microns in diameter (the refractive index is approx. 1.39). The optical energy of the 400 microns droplet was found to be focused in a very narrow angle at the forward direction (near $0^{\circ}$ ). By placing the camera sensor in parallel with the laser sheet, the camera sensor receives the light energy scattered at $90^{\circ}$, which reduces the possibility of sensor saturation. Moving the view angle slightly towards the forward scattering direction (at approx. $80^{\circ}-85^{\circ}$ instead of $90^{\circ}$ ) was found to produce a clear image of the internal reflections in the fluid droplet.

\section{Laser-Sheet Imaging Method}

Microdroplets are usually considered to be entirely located within a uniform lightsheet when the light sheet is much wider than the droplet size. This paper investigates the effect of a droplet relative location within the laser sheet on the light intensity profile of its image. When an infinitely thin light sheet crosses a spherical droplet, the optical energy received by the photosensors is dependent on the droplet location to the light sheet. In reality, the optical energy of a Gaussian light sheet gradually reduces when moving away from the centre of the sheet pattern, and the sheet waist becomes wider by moving further from the focal point of the sheet optics. The light sheet in this experiment was generated using a $(100 \mathrm{~mm})$ cylindrical lens, as shown in Figure 2. The effective width of the laser sheet in this case 
was approximately $3 \mathrm{~mm}$ at the object plane, which is enough to detect a droplet location within the sheet pattern. An $\mathrm{Nd}$ : YAG green laser $(532 \mathrm{~nm})$ was used for illumination, providing $600 \mathrm{~mJ}$ of optical power during a short pulse width of 5 ns. A CCD camera (PCO2000, $2048 \times 2048$ pixels) was used for digital imaging, using a spatial resolution of 32.5 micrometer per pixel. The experimental rig design was previously reported in more details in [8]. JP8 aviation fuel (kerosene) was injected using a pressure-swirl nozzle approximately 584 microns in diameter, fitted on an IWP023 gasoline injector. The injector was operated at (0.5-1) MPa injection pressure and $(0.3-3) \mathrm{kHz}$ injection frequency. A metallic aperture was attached to the cylindrical lens in order to keep the Gaussian effect to only one side of the laser sheet.

\section{Experimental Observations}

Fuel droplets, with diameters larger than 200 times the laser's wavelength, were detected in great quantity during our experiments on fuel injector systems equipped with heating matrixes. A large number of particles condensed on the heater grid, forming giant droplets, which were later released with the flow. For small droplets $(<100$ microns), only droplets located inside the light sheet area and within the sensor sensitivity region were detected. For large droplets, several illumination patterns and light intensity levels were identified.

Large spheres are often dealt with in literature as a special type of optics, since light diffraction is not the only dominant effect in this case. When a light beam encounters an obstacle, the obstacle scatters the light energy into different angles. The term "light scattering" describes a number of mechanisms in which a droplet/particulate emits electromagnetic waves as a secondary source of energy. For particles with a certain degree of transparency, the refracted light through the particle generates secondary scatters, in addition to the primary edge scatters, due to a set of internal reflections inside that particle (see [9] for more details). Beside the light diffraction and interreflections, large particles show a big deal of light reflection off the external surface (Figure 3 ). The latter effect becomes much more dominant in particles much larger than the wavelength, and for high refractive indices. Two effects appear in this case [6]; the first is called the "edge phenomena," where fields distribute near the edges causing an increment in the forward scattering. The second effect is called the "surface wave" phenomena where the light waves spread (or "creep") to the dark side of the droplet. These two effects were observed and imaged in the current work. For a droplet image not completely saturated on the CCD, it was noticed that a single interreflection point appeared inside it, sending the light rays backward towards the source, and contributing to the overall backscattering. This effect is referred to in the literature as the "glory effect" [10], which is responsible for the rainbow phenomenon in nature. These factors together contribute in the illumination of the droplet surface and, therefore, assist in identifying the droplet size and shape.

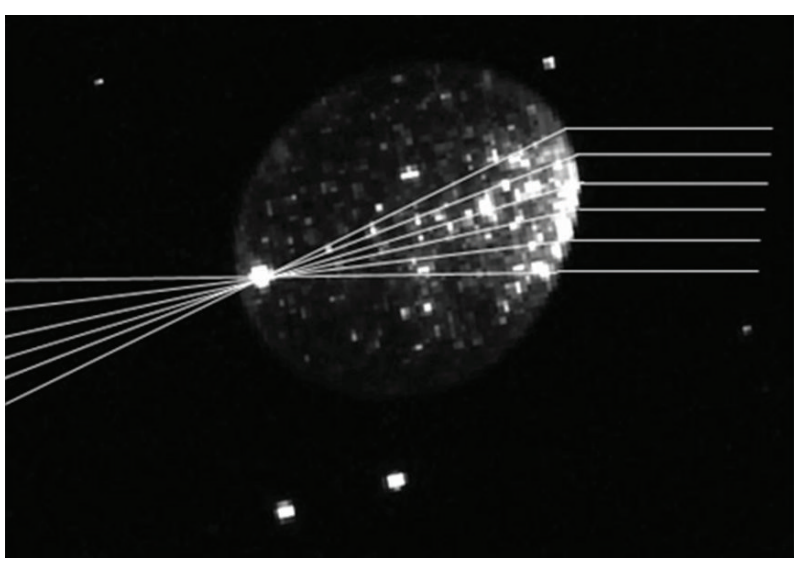

Figure 3: Kerosene droplet (approx. $2.2 \mathrm{~mm}$ in diameter) photograph; demonstrating the light vectors behaviour inside a fluid droplet (in air).

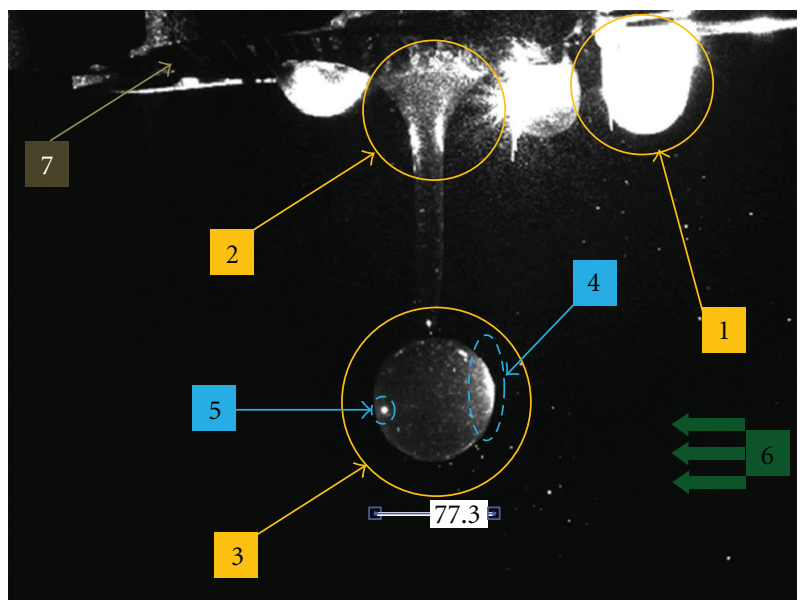

FIGURE 4: Image of JP8 fuel droplets, showing the process of a large droplet formation (laser pulse duration $5 \mathrm{~ns}$ ) during the resting phase of the injection incident (1.2 ms). (1) A droplet attached to the heating matrix located at the saturation region (in the middle) of the laser sheet. (2) A developing droplet (ligament) at the side of the laser sheet; this part is affected by the secondary reflections from back droplets. (3) A completely developed droplet (around $2.51 \mathrm{~mm}$ in diameter) at the side laser sheet. (4) The main reflection on the surface where the laser rays strike the droplet. (5) Interreflection where the light rays leave the droplet after being deflected inside the droplet. (6) The direction of the incident laser beams. (7) The body of the injector and the heating matrix.

\section{Data Analysis}

By taking an image of the injector exit at the resting phase of the injection cycle (Figure 4), it was possible to distinguish between three different cases in terms of a fluid droplet location in relation to the light sheet.

(a) A droplet entirely outside the light sheet region: this was not detected by the CCD.

(b) A droplet entirely inside the light sheet region: this showed high intensity light scattering pattern 


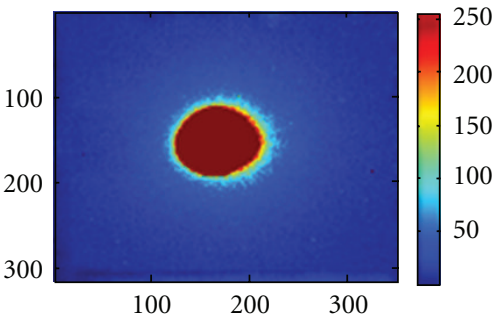

(a)

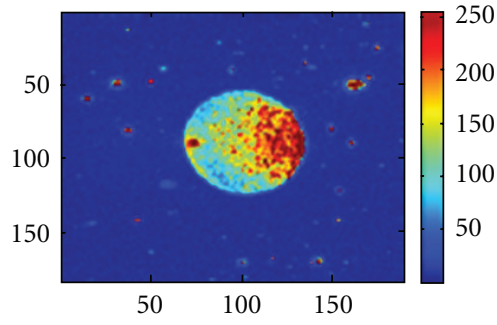

(b)

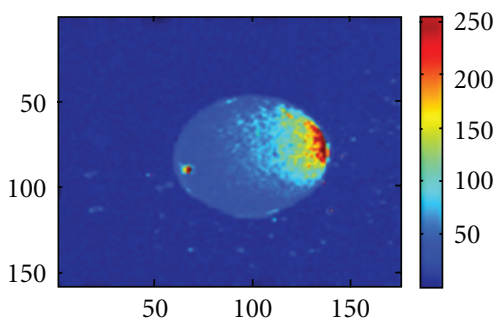

(c)

FIgURE 5: Light intensity distribution for fuel droplets (close in size) at different relative positions from the centre of the light sheet.

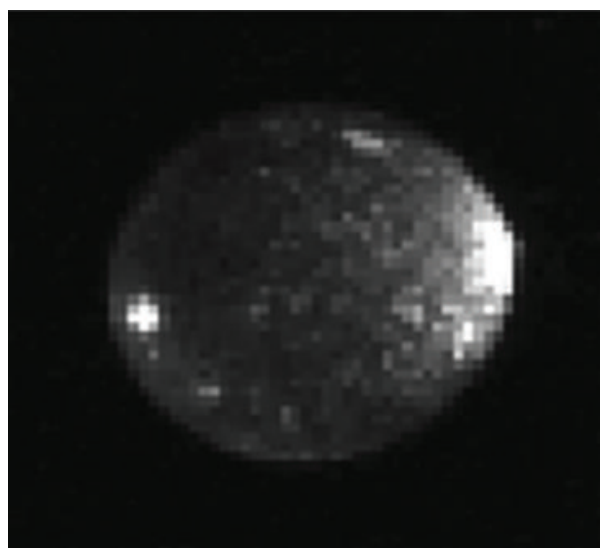

(a)

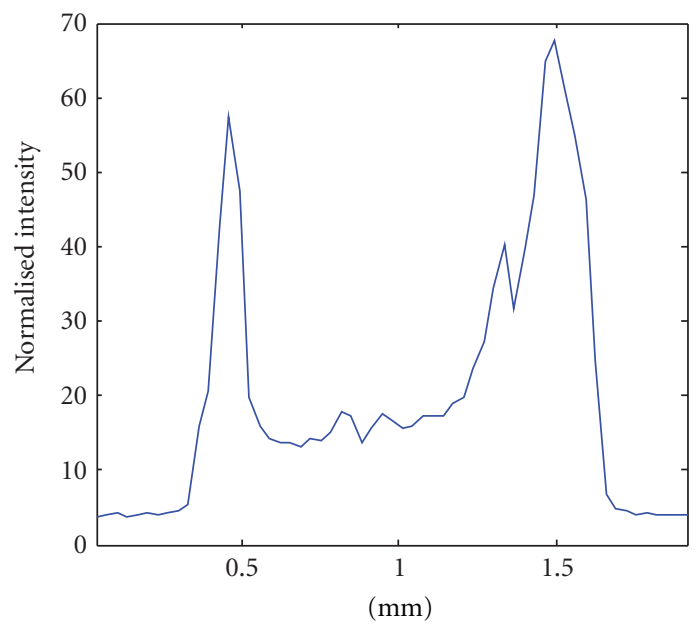

(c)

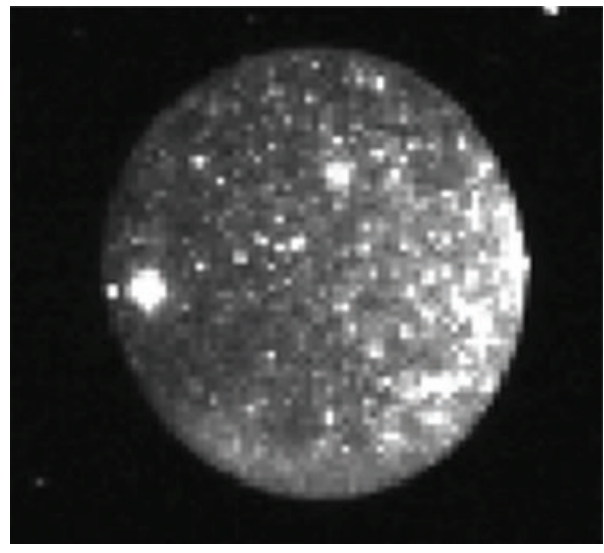

(b)

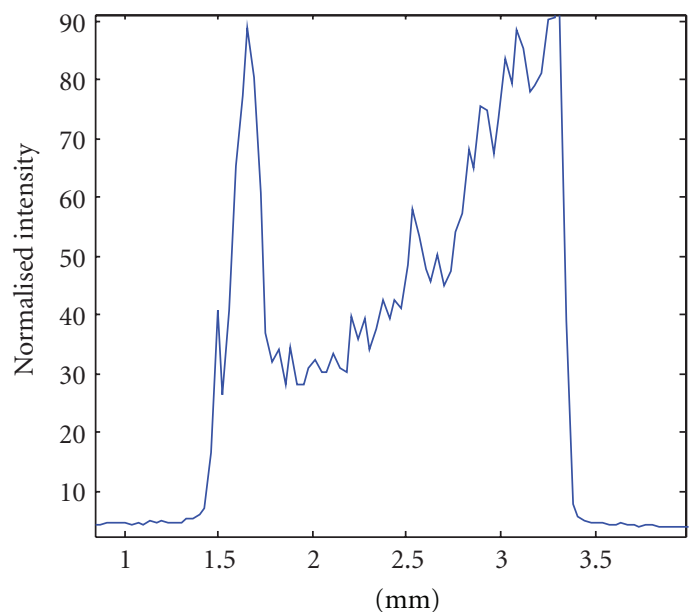

(d)

FIgURE 6: Intensity profile (normalised to maximum) of fuel droplets $(1.4,2.1) \mathrm{mm}$ in diameter.

around the droplet, besides a full illumination of the droplet surface. The received light energy by the CCD increased closer to the centre of the beam pattern. The droplet image could be saturated, and the droplet contours were not clearly recognised because of the corona around its surface. The corona effect increased as the droplet diameter decreased.

(c) A partly illuminated droplet: in this case a droplet scattering pattern was found to be dependent on the droplet location within the light sheet at the radial axis (the third dimension).

The scattering cross-section of a droplet $A_{s}$ represents the amount of energy carried by the incident wave falling on a certain area, which is given by

$$
I_{\theta}=I_{0} \frac{1}{R^{2}} A_{s},
$$

where $R$ is the distance between the secondary source and the observer. The ratio between the scattering cross-section and 
the droplet (geometrical) cross-section is called the scattering efficiency (see Figure 1), which is given by

$$
Q_{s}=\frac{A_{s}}{A_{d}}=\frac{S^{2}}{k^{2}}
$$

where $(S)$ is the amplitude function in Mie solution and $(k)$ is the wave number.

If a particle is illuminated by a plane wave light beam, the scattering efficiency is theoretically not affected by the droplet location within that beam, which is an acceptable assumption for very small droplets to the beam waist. For large droplets illuminated by a Gaussian beam, a modified theory, called Generalized Lorenz-Mie theory (GLMT), has been developed [11], where a new coefficient has been added to the amplitude function series. This coefficient is dependent on the beam waist radius $\left(R_{w}\right)$ and the wavelength of the source $\lambda$ as follows:

$$
S_{i} \propto e^{\left[-(i+0.5) \lambda / 2 \pi R_{w}\right]^{2}} .
$$

A droplet location to the central beam determines the effective geometrical cross-section $\left(A_{d}=\pi r^{2}\right)$ and consequently affects the diffraction efficiency of that droplet for all angles of observation, including particles located at the side of the beam where the optical the intense.

\section{Droplet Displacement Calculation}

The proposed method for droplet displacement calculation at $Z$-axis with respect to the central laser beam relies on finding the relation between the overall light intensity of a given droplet image and the radius of that droplet. The droplets which are entirely illuminated by the central beam were used for calibration, where the droplet diameter can be extracted directly from the binary image using a suitable threshold and edge detection algorithm. The light intensity was numerically defined by the DC coefficient of a two-dimensional discrete cosine transform (2D-DCT) for each droplet sample. The DCT is popular in image and video compression applications. The DC coefficient of the transformed image contains the "image energy" and corresponds to the overall light intensity received by the camera sensor. The DC coefficient is associated with the average brightness of the entire image matrix, while the rest of the AC coefficients depend on the deviations from that average.

When a particle moves away from the central beam, the relation $\mathrm{DC}=f(r)$ deviates from the calibration value, and what can be calculate in this case is a virtual radius $\left(r_{s}\right)$ associated with the new DC coefficient value $\left(\mathrm{DC}_{s}\right)$. The radius $\left(r_{s}\right)$ represents the cross-section of a sphere which produces a brightness coefficient that equals to $\mathrm{DC}_{s}$, when the droplet is located in the centre of the beam. The advantage of the droplet large size is that the actual size can be calculated directly from the acquired images in all cases because of the "edge" and "surface wave" phenomena as explained earlier. Because the fluid droplets are not always perfectly spherical, the equivalent spherical diameter (ESD) method is used [12], where a particle volume is

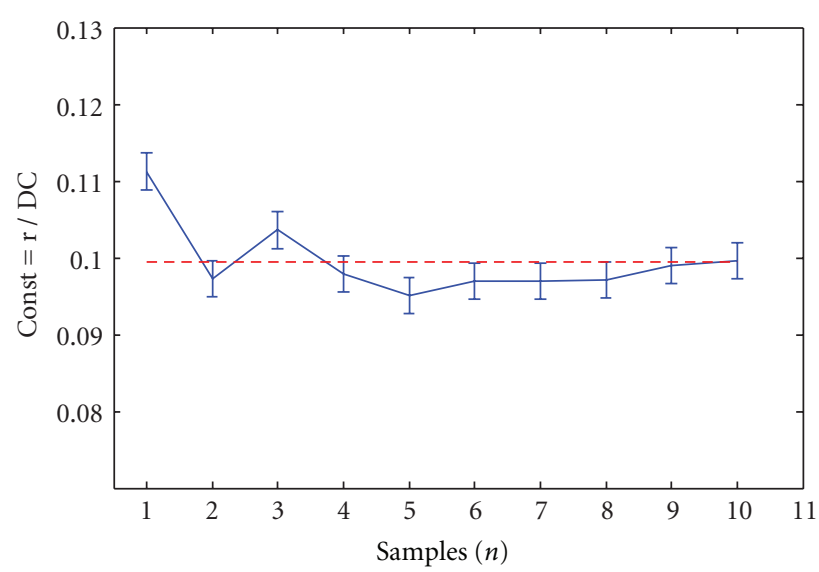

Figure 7: Samples of large particles in the middle laser beam with the corresponding binary image. The graph shows the calculation of the ratio $\mathrm{r} / \mathrm{DC}$ in the calibration step using the same samples showing above.

measured, and then linked to the diameter of a sphere which has the same volume. Thereby, the displacement $(d)$ of a particle from the beam centre can be estimated through the difference between the cross-section radius $\left(r_{s}\right)$ and the actual radius $(r)$ of the particle.

\section{Results and Discussion}

The generated images showed that the light intensity profile varies from one droplet to another even when they are similar in size. The light intensity level was found dependent on the relative position of the droplet to the centre of the Gaussian beam (Figure 5). The corona effect was apparent when for droplets entirely located within the high power centre of the beam, and the backscattering reduced for droplets located at the sides of the laser sheet.

Droplets partly covered by the central beam were not saturated, and thereby it was possible to spot their structure and homogeneity. By processing the images, it was possible to identify the internal reflections inside individual fuel droplets (Figure 6) and calculate the light intensity distribution profile on a droplet surface.

Fluid droplets act as miniature convex lens, and the light dispersion mechanism is dependent on the refractive index of the fluid material. It was empirically found that the ratio between a droplet radius and its image DC coefficient is constant for all particles located within the central laser beam:

$$
\text { Const }=\frac{r(\mu \mathrm{m})}{\mathrm{DC}} \text {. }
$$

This constant was firstly calculated for calibration using droplets in the central laser beam, and it was equal to 0.0995 in our example, with a standard deviation of $4.7 \%$, as shown in Figure 7. Another set of data was then selected where large droplets were deviated from the central laser beam. The actual radii $(r)$ were directly calculated from the digital images using an edge detection technique. The (DCT) 

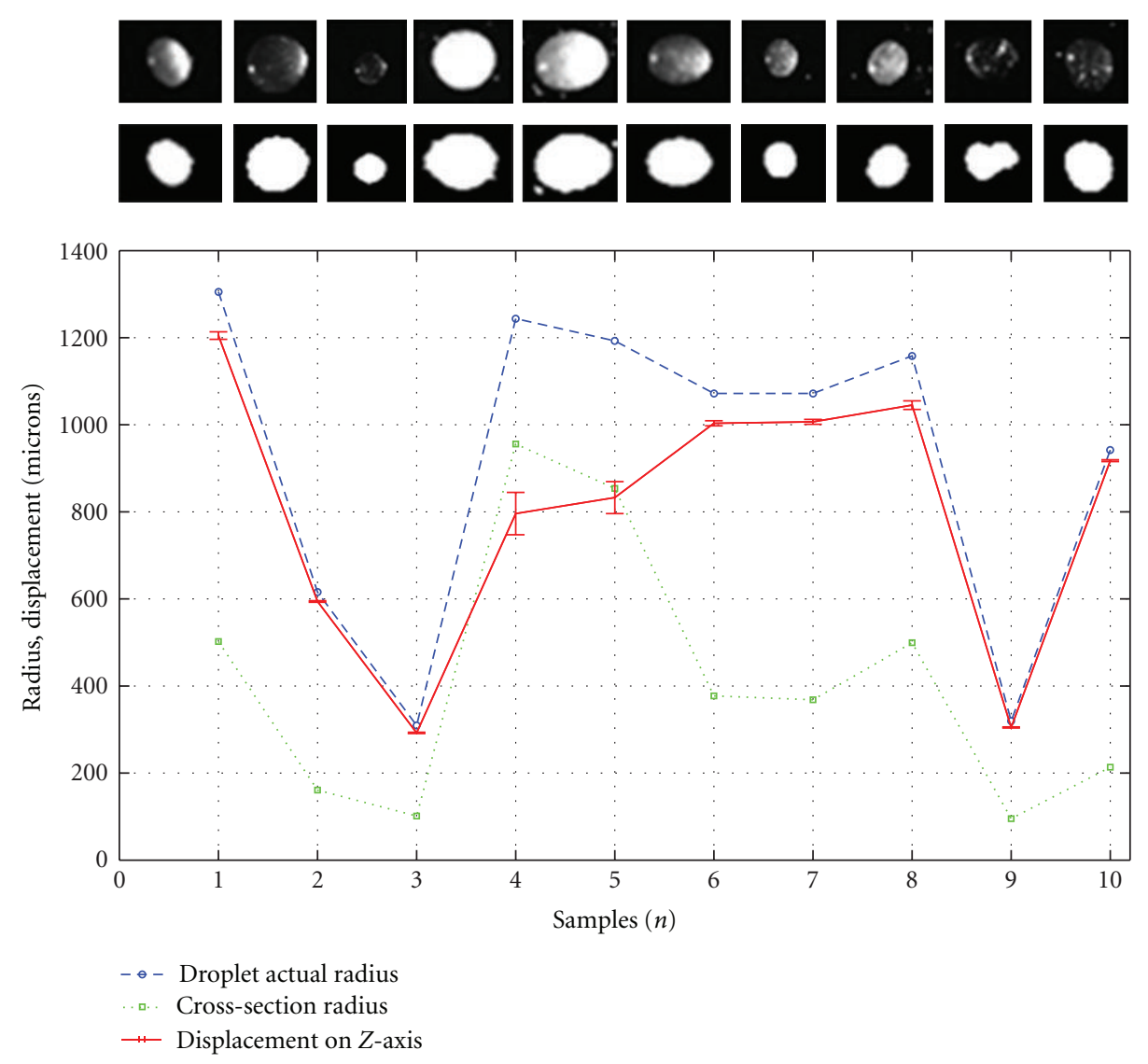

FIGURE 8: Large kerosene droplets and their radii with the estimation of displacement at the third dimension of the FOV ( $Z$-axis).

coefficients $\left(\mathrm{DC}_{s}\right)$ by the new set of data are associated with the illuminated part of the droplets. It was found that these coefficients are higher for droplets closer to the Gaussian beam's centre. A new set of radii was then calculated for spheres that reflect the same amount of light, that is, producing the same (DC) coefficients, as the illuminated cross-sections of these drops. The new virtual radius of each droplet is calculated using the relation:

$$
\text { Const }=\frac{r}{\mathrm{DC}}=\frac{r_{s}}{\mathrm{DC}_{s}} \text {. }
$$

Then, the displacement $(d)$ from the central beam can be calculated from

$$
d=\sqrt{r^{2}-r_{s}^{2}}
$$

Figure 8 shows samples of the fuel droplets at different positions (on $Z$-axis), and the extracted binary images. The graph illustrates the droplet calculated radius, the crosssection radius, and the estimated displacement from the centre of the Gaussian beam in microns, showing the error produced only by the deviation in the constant (Const) value.

The majority of the nonspherical droplets were located within the first $2 \mathrm{~cm}$ downstream of the nozzle exit due to the high kinetic energy gained during the pressurising stage. Smaller droplets lose a big deal of their energy earlier

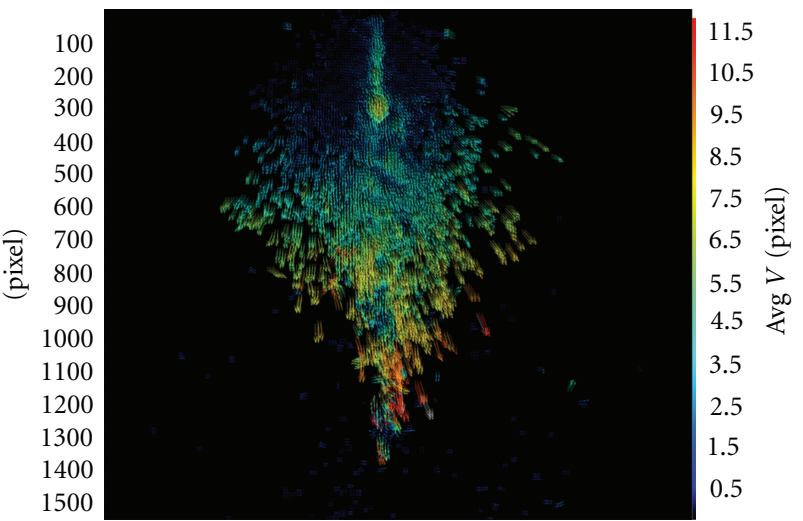

Figure 9: PIV of kerosene spray at $0.5 \mathrm{MPa}$ pressure (mean velocity). Pulse width $=2 \mathrm{~ms}$, Separation between double pulses = $15 \mu \mathrm{s}$, resolution $=52 \mu \mathrm{m} /$ pixel $(1$ pixel displacement $=3.47 \mathrm{~m} / \mathrm{s})$; measured at $2 \mathrm{~ms}$ after start of injection.

than the bigger droplets. Figure 9 shows the velocity vector field of the fuel spray, generated by applying particle Image velocimetry (2D-PIV) using 15 microseconds time delay between the double frames. By the end of the $2 \mathrm{~ms}$ injectioncycle duration, the average velocity of the central jet was found to be between 9 and $10 \mathrm{~ms}^{-1}$. Therefore, only droplets 
bigger than $2 \mathrm{~mm}$ are able to exceed the critical Weber number $\left(\mathrm{We}_{c}=10-12\right)$, which eventually deform and split up into smaller droplets. At earlier stages of atomisation, the discharge velocity of the fluid jet was much higher (approximately $50 \mathrm{~m} / \mathrm{s}$ ), which allowed even smaller droplet to deform and split up. Smaller droplets have a relatively small Weber number and they maintain their spherical shape for longer time. The low velocity of the small droplets (smaller than 100 microns) maintains them floating in the upper side of the spray jet, giving the spray its cone-like shape, while the unbroken large droplets travel faster towards the spray peripheries.

\section{Conclusion}

It is possible to perform a dynamics analysis of high-speed fluid droplets much larger than the wavelength using pulsed lasers and digital imaging techniques. For large droplets, reflections generated by the "edge" and "surface wave" phenomena become more dominant than the light corona generated by the light diffraction effect, especially at wide angles of observation (such as $90^{\circ}$ ). This improves the illumination of the droplet surface at the shadow region and provides the ability to investigate the droplet profile and calculate its diameter directly from the binary image. Large fluid droplets in a Gaussian-shaped light sheet can be considered partly located in the central beam, and, therefore, the surface structure of a particle can be detected by avoiding the saturation of the camera sensor. The relative location of a particle to the central light beam is directly connected to the light energy received by the photosensor. This relation can be used to estimate a particle position on the third dimension ( $Z$ axis) using $2 \mathrm{D}$ images, and possibly the velocity at this direction using double-exposure images. However, there is a certain element of uncertainty involved when calculating the particle diameter to light intensity ratio because of the nonperfect spherical shape of the particles on one hand, and the nonnegligible width of the central waist of the laser beam (where the camera sensor is saturated) on the another hand. Furthermore, the limited dynamic range of the CCD sensor leads to an unavoidable saturation of some parts of the droplet's image occasionally. In order to apply on smaller particles with high accuracy, a very thin light sheet should be produced, where the changes in the light energy throughout the Gaussian profile of the sheet are distinguishable on the camera sensor. The droplet velocity distribution is presented for the global spray using image cross-correlation-based PIV technique. This is used to predict the spray regions where the fluid droplets are likely to encounter deformation (from the spherical shape) and a possible further (secondary) atomisation during the short injection incident, depending on the Weber number, which is determined by the droplet mean diameter and velocity.

\section{Abbreviations}

CCD: Charge-coupled device

DCT: Discrete cosine transform

ESD: Equivalent spherical diameter
FOV: $\quad$ Field of view

GLMT: Generalized lorenz-Mie theory

Nd-YAG: Neodymium-doped yttrium aluminium garnet

PDA: Phase doppler anemometry

PIV: $\quad$ Particle image velocimetry

We: Weber number.

\section{Nomenclature}

$\rho_{g}$ : Gas density

$U$ : Liquid discharge velocity

$D$ : Droplet diameter

$\sigma:$ Surface tension

$A_{s}$ : Scattering cross-section

$A_{d}$ : Droplet cross-section

$R$ : Distance between object and observer

I: Light intensity

$Q_{s}$ : Scattering efficiency

$k$ : Wave number

$\lambda$ : Wavelength

$S: \quad$ Mie amplitude function

$r$ : Radius (of a droplet).

\section{References}

[1] D. R. Guildenbecher et al., "Droplet deformation and breakup," in Handbook of Atomization and Sprays; Theory and Applications, N. Ashgriz, Ed., Springer, New York, NY, USA, 2011.

[2] R. I. Nigmatulin, Dynamics of Multiphase Media, vol. 1, Hemisphere Publishing, 1991.

[3] M. G. Pai, I. Bermejo-Moreno, O. Desjardins, and H. Pitsch, "Role of Weber number in primary breakup of turbulent liquid jets in crossflow," Center for Turbulence Research Annual Research Briefs 2009, pp. 145-158, 2009.

[4] R. Morgan, J. Wray, D. A. Kennaird, C. Crua, and M. Heikal, "The influence of injector parameters on the formation and break-up of a diesel spray," SAE Transactions Journal of Engines, vol. 110, no. 3, pp. 389-399, 2002.

[5] C. T. Crowe, M. Sommerfeld, and Y. Tsuji, Multiphase Flows with Droplets and Particles, CRC Press, New York, NY, USA, 1998.

[6] H. C. van de Hulst, Light Scattering by Small Particles, Dover, New York, NY, USA, 1981.

[7] C. Godefroy and M. Adjouadi, "Particle sizing in a flow environment using light scattering patterns," Particle and Particle Systems Characterization, vol. 17, no. 2, pp. 47-55, 2000.

[8] R. Zakaria, P. Bryanston-Cross, and S. Addy, "Optical diagnostics in high-speed fuel spray; methodology and analysis," in Proceedings of the 1st International Conference on Digital Object Identifier (ICP '10), pp. 1-6, 2010.

[9] P. Laven, "Separating diffraction from scattering: the milliondollar challenge," Journal of Nanophotonics, vol. 4, no. 1, Article ID 041593, 2010.

[10] S. Solimeno, B. Crosignani, and P. D. Porto, Guiding, Diffraction, and Confinement of Optical Radiation, Chapter 5, Academic Press, New York, NY, USA, 1986.

[11] G. Grehan, G. Gouesbet, and F. Guilloteau, "Comparison of the diffraction theory and the generalized Lorenz-Mie theory for a sphere arbitrarily located into a laser beam," Optics Communications, vol. 90, no. 1-3, pp. 1-6, 1992. 
[12] J. T. Kashdan, J. S. Shrimpton, and A. Whybrew, "A digital image analysis technique for quantitative characterisation of high-speed sprays," Optics and Lasers in Engineering, vol. 45, no. 1, pp. 106-115, 2007. 

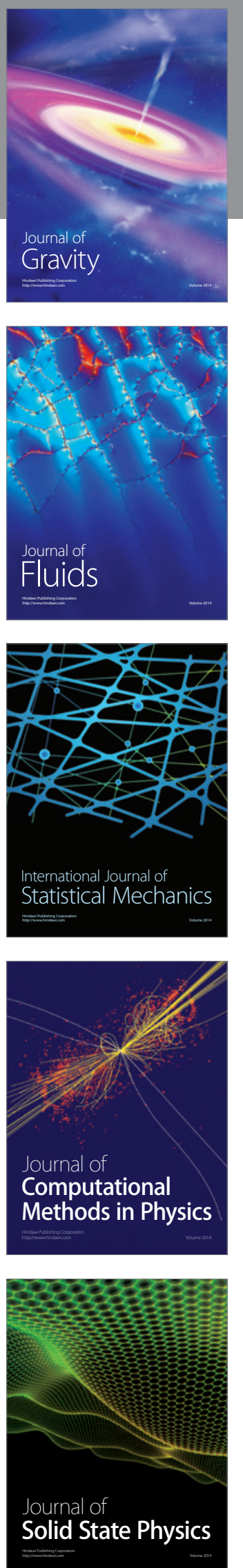
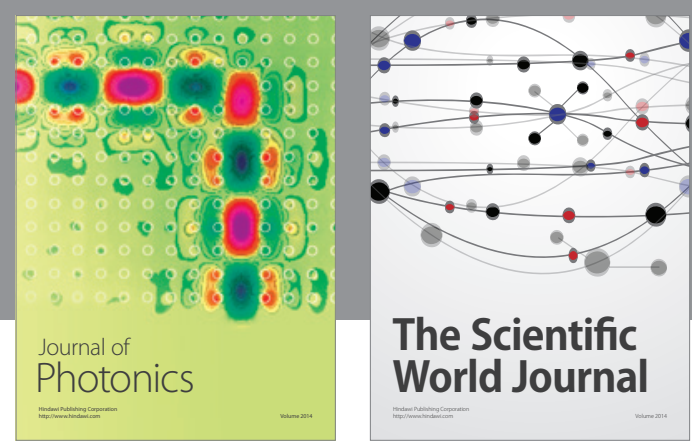

The Scientific World Journal

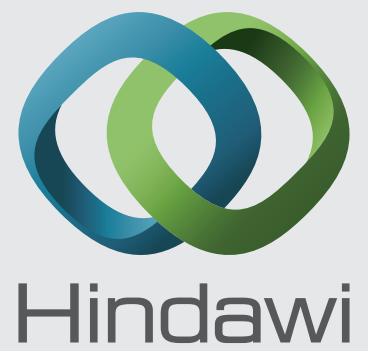

Submit your manuscripts at http://www.hindawi.com
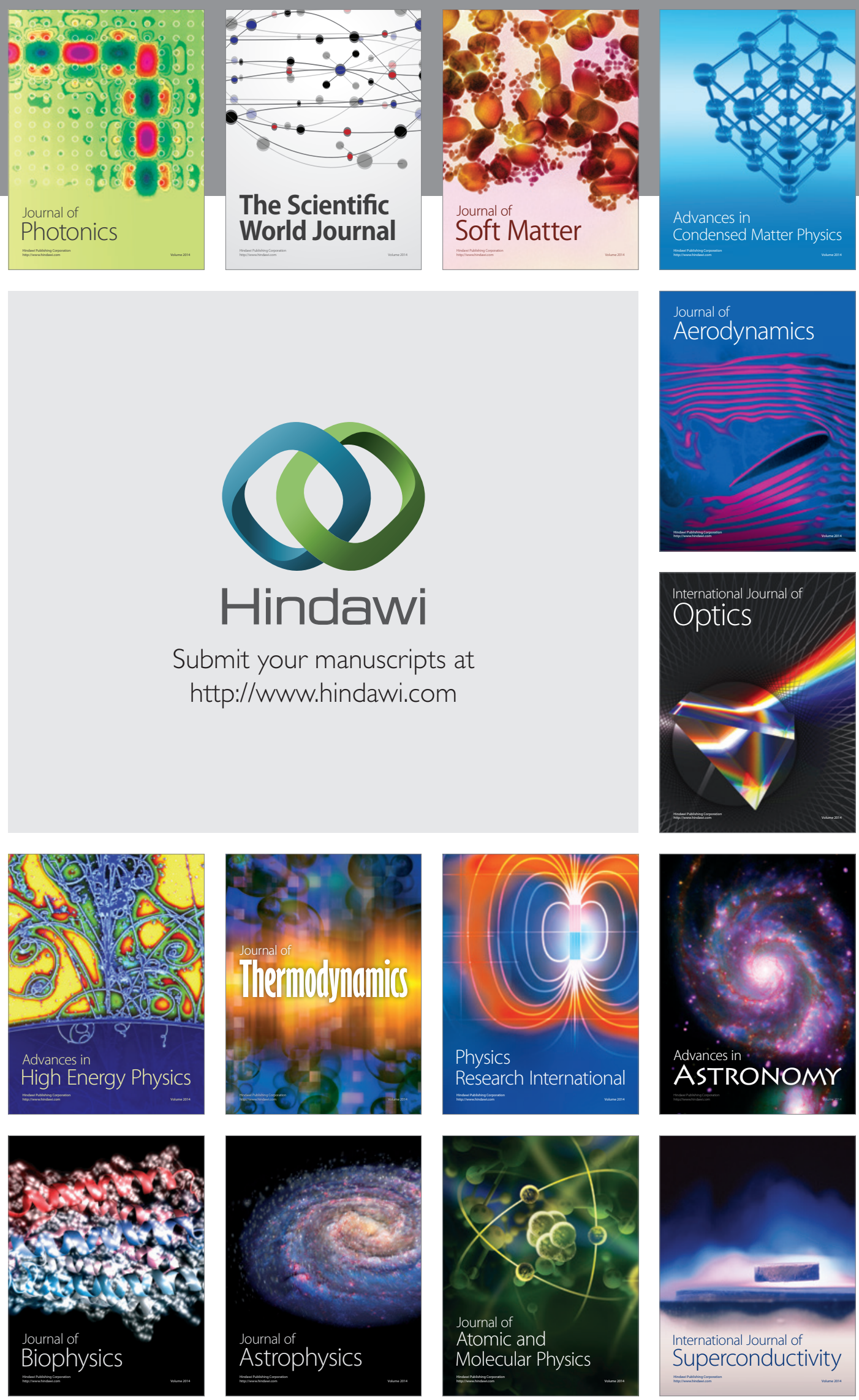
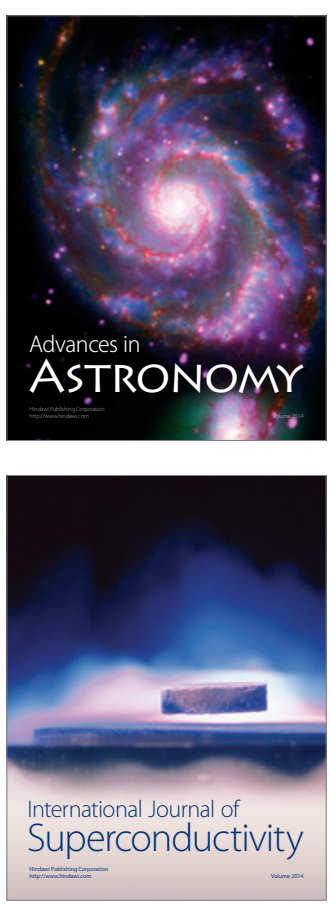